\title{
Construction of an miRNA-regulated drug-pathway network reveals drug repurposing candidates for myasthenia gravis
}

\author{
YUZE CAO $^{1,2^{*}}$, XIAOYAN LU ${ }^{1 *}$, JIANJIAN WANG $^{1}$, HUIXUE ZHANG $^{1}$, ZHAOJUN LIU $^{1}$, SI XU ${ }^{1}$, \\ TIANFENG WANG ${ }^{1}$, SHANGWEI NING $^{3}$, BO XIAO $^{2}$ and LIHUA WANG ${ }^{1}$ \\ ${ }^{1}$ Department of Neurology, The Second Affiliated Hospital, Harbin Medical University, Harbin, \\ Heilongjiang 150081; ${ }^{2}$ Department of Neurology, Xiangya Hospital, Central South University, Changsha, Hunan 410008; \\ ${ }^{3}$ College of Bioinformatics Science and Technology, Harbin Medical University, Harbin, Heilongjiang 150081, P.R. China
}

Received February 26, 2016; Accepted January 4, 2017

DOI: $10.3892 /$ ijmm.2017.2853

\begin{abstract}
Myasthenia gravis (MG) is a rare debilitating autoimmune neuromuscular disorder. Many studies have focused on the mechanism and treatment strategies of MG. However, the exact pathogenesis of MG and effective treatment strategies remain unclear. Recent studies have indicated that microRNAs (miRNAs or miRs) can regulate the pathological pathways of MG, suggesting their potential role in novel treatments. In the present study, we created a comprehensive catalog of experimentally confirmed MG risk genes and miRNAs by manually mining published literature and public databases. Based on these genes and miRNAs, we identified 41 MG risk pathways and 105 approved drugs that can affect these pathways. Some important MG-related pathways, such as hsa04060 (cytokinecytokine receptor interaction) and hsa05200 (pathway in cancer), were found to be regulated by MG risk miRNAs and drugs. Furthermore, we constructed an miRNA-regulated drug-pathway network and identified miRNAs and drugs that synergistically regulate key MG pathways and biological processes. We developed a drug repurposing strategy to identify 25 drug repurposing candidates for MG; several of these drugs, such as rituximab, adalimumab, sunitinib, and muromonab, have the potential to be novel MG treatment drugs. This study provides novel insight into the pathogenesis of MG and potential drug candidates for MG were identified.
\end{abstract}

Correspondence to: Professor Lihua Wang, Department of Neurology, The Second Affiliated Hospital, Harbin Medical University, Harbin, Heilongjiang 150081, P.R. China

E-mail: wanglh211@163.com

Dr Bo Xiao, Department of Neurology, Xiangya Hospital, Central South University, Changsha, Hunan 410008, P.R. China

E-mail: xiaobo_xy@126.com

*Contributed equally

Key words: myasthenia gravis, miRNA, pathway, drug-pathway network, drug repurposing candidates

\section{Introduction}

Myasthenia gravis (MG) is a rare antibody-mediated autoimmune disease characterized by muscle fatigue manifested as ptosis, diplopia, dysarthria, dysphagia and limb weakness $(1,2)$. Researchers have elucidated important mechanisms underlying MG (3). Current treatment strategies for MG mainly include cholinesterase inhibitors, immune-suppressants and thymectomy (4). However, various common side effects are major obstacles to the effective use of these drugs.

In recent years, many studies have revealed that microRNAs (miRNAs or miRs) are important regulators of MG pathogenesis. For example, miR-320a targets mitogen-activated protein kinase 1 (MAPK1) and regulates COX-2 expression through the ERK/nuclear factor- $\kappa \mathrm{B}(\mathrm{NF}-\kappa \mathrm{B})$ pathway (5). Inhibition of miR-146a was found to reduce cell surface costimulatory molecules, such as CD40, CD80, CD86 and intracellular TLR4 and NF- $\kappa$ B levels in AChR-specific B cells (6). miR-181c was found to negatively regulate the production of proinflammatory cytokines interleukin-7 (IL-7) and IL-17 (7). Recently, as new potential therapeutic targets, miRNAs have attracted increased interest (8). For example, as miR-155 is involved in inflammation and immune diseases (9), therapies targeting miR-155 may be applicable to various autoimmune and inflammatory disorders. These findings indicate that miRNAs have potential influence on the pathways underlying MG pathogenesis and can thus affect the treatment of MG. However, most of these studies have focused on only one or a few miRNAs in cell lines or using limited samples.

The research and development of new drugs for MG is a time-consuming and labor-exhausting process. In recent years, drug repurposing has been a vital part of the drug discovery process that can uncover new indications for existing drugs (10). For example, Hu and Agarwal constructed a large scale disease-drug network for effective drug repositioning (11). One of our previous studies constructed a small molecule and miRNA association network for Alzheimer's disease (AD) (12). Ye et al proposed a disease-oriented strategy for evaluating the relationship between drugs and diseases based on their pathway profile (13). These studies are critical for drug discovery and development in future research. However, few studies have focused on drug repurposing for MG $(14,15)$. 
In the present study, we identified the risk pathways regulated by MG risk genes, miRNAs and drugs and constructed an miRNA-regulated drug-pathway network (MDPN). We analyzed the properties of the MDPN and determined how the miRNAs and drugs synergistically regulate MG risk pathways. Moreover, we identified drug repurposing candidates for MG and clarified their potential mechanisms as novel treatment drugs. Our results may provide a novel viewpoint with respect to the mechanism and treatment of MG.

\section{Materials and methods}

Human MG risk gene data. We manually read literature published before June 1, 2015 by searching the PubMed database using the terms [myasthenia gravis (MeSH Terms) and English (Language)]. The species of the risk genes was limited to 'Homo sapiens'. We thoroughly read the 9,474 items returned by our searches and selected MG risk genes that met the following standards oulined in a previous study (16): i) the gene was presented in at least 5 MG samples (including blood samples and thymic tissue samples); ii) the gene was detected using reliable experimental methods, such as microarrays and RT-PCR; and iii) the gene was significantly differentially expressed (mRNA level or protein level). In addition, we also collected MG risk genes from current public databases, including the Genetic Association Database (GAD) (17), DisGeNET (18), Online Mendelian Inheritance in Man (OMIM) (19) and Functional Disease Ontology Annotation (FunDO) (20).

$M G$ risk miRNAs and miRNA targets. Similar to the collection of MG risk genes, we also manually collected MG risk miRNAs from different sources, including the Human microRNA Disease Database (HMDD v2.0) (21), PhenomiR (22), and a literature search using the keywords 'miRNA' and 'myasthenia gravis' or 'microRNA' and 'myasthenia gravis' or 'miR' and 'myasthenia gravis' in PubMed. Furthermore, we downloaded the target genes of these MG risk miRNAs from five experimentally validated miRNA target databases, including miRWalk (23), miRTarBase (24), miR2Disease (25), miRecord (26) and miRSel (27).

Human drug target data. Drugs and their target genes were downloaded from DrugBank (version 4.0) (28), with the species of the drug targets restricted to 'Homo sapiens'. Drugs with less than 5 target genes were excluded from this study.

Pathway analysis. The physiological pathways implicated in $\mathrm{MG}$ were determined through the functional enrichment of significant gene lists using the Database for Annotation, Visualization, and Integrated Discovery (DAVID) (29). The Kyoto Encyclopedia of Genes and Genomes (KEGG) pathway enrichment of the MG risk gene lists was calculated using a P-value cutoff of 0.05 (Fisher's exact test). In addition, the miRNA-related pathways and drug-related pathways were obtained using their target gene sets. Then, we constructed an MDPN consisting of drugs, miRNAs and their common MG risk pathways. Cytoscape 2.8.3 was utilized to visualize the network, and the network properties were analyzed using the Network Analysis plugin (30). Gene ontology (GO) biological process enrichment was performed using $\mathrm{GO}$ level 3 (FDR <0.05).

Association scores (AS) and significance levels between drugs and $M G$. Our drug repurposing strategy was developed based on two biological hypotheses: first, disease risk genes do not exert their functions independently but rather through regulating special pathways. Second, drugs exert therapeutic effects by regulating pathways involved in disease pathology rather than by directly targeting disease-associated genes. Drug pathways and MG risk pathways were obtained from the MDPN network. We calculated AS between the drugs and MG as described by the following formula (13):

$$
S_{\text {drugi }, M G}=-\lg \sum \sqrt{p_{M G, \mathrm{k}} \times p_{\text {drugi }, k}}
$$

Here, $p_{M G, k}$ is the enriched P-value of MG on pathway ' $k$ '; $p_{\text {drugi, }}$ is the enriched P-value of drug ' $i$ ' on pathway ' $k$ '; $k$ represents the pathway that is most significantly affected by both MG risk genes and drug ' $i$ ' targets.

Furthermore, to evaluate the specificity of the association between the drugs and MG, we performed random permutations of the pathways and calculated the $\mathrm{Z}$ scores of the drugs and MG. While retaining the pathways of the drugs, random pathway profiles of the drugs were generated by randomly sorting the pathway 10,000 times. For each random profile, $S_{\text {random,drugi }}$ was calculated according to formula (1). In this study, the Z-score was used to evaluate the specificity of the association between the drug and disease:

$$
Z_{\text {drugi,MG }}=\frac{S_{\text {drugi,MG }}-\text { average }\left(S_{\text {random,drugi }}\right)}{\operatorname{std}\left(S_{\text {random,drugi } i}\right)}
$$

where the average $\left(S_{\text {random,drugi }}\right)$ is the average association score between random cases and drug $i$, and std $\left(S_{\text {random,drugi }}\right)$ is the standard variation of association between random cases and drug $i$.

\section{Results}

A comprehensive catalog of MG risk genes, miRNAs and pathways. We created a comprehensive catalog of 162 risk genes that have been experimentally confirmed to be associated with MG. Among these genes, 123 risk genes were manually collected by literature mining, and 39 risk genes were compiled from public databases. Based on these risk genes, we identified $45 \mathrm{MG}$ risk pathways $(\mathrm{P}<0.05)$ using KEGG enrichment analysis. The top 25 pathways and their P-values are shown in Fig. 1A. According to the pathway classification of the KEGG database, all pathways were divided into seven categories (Fig. 1B). We found that most of the MG risk pathways are related to 'Human Diseases: Immune diseases', 'Organismal Systems: Immune system' and 'Human Diseases: Cancers', which implies that we can use immune pathways as well as cancerous pathways to functionally characterize MG. As a paraneoplastic phenomenon, MG is mainly caused by thymoma but can also be associated with other cancers, such as renal cell carcinoma (31). More importantly, hsa04060 (cytokine-cytokine receptor interaction) was the first significantly enriched pathway; $39 \mathrm{MG}$ risk genes participated in this pathway, which plays key roles in MG (Fig. 1C). We also performed a functional enrichment analysis of these risk genes, 

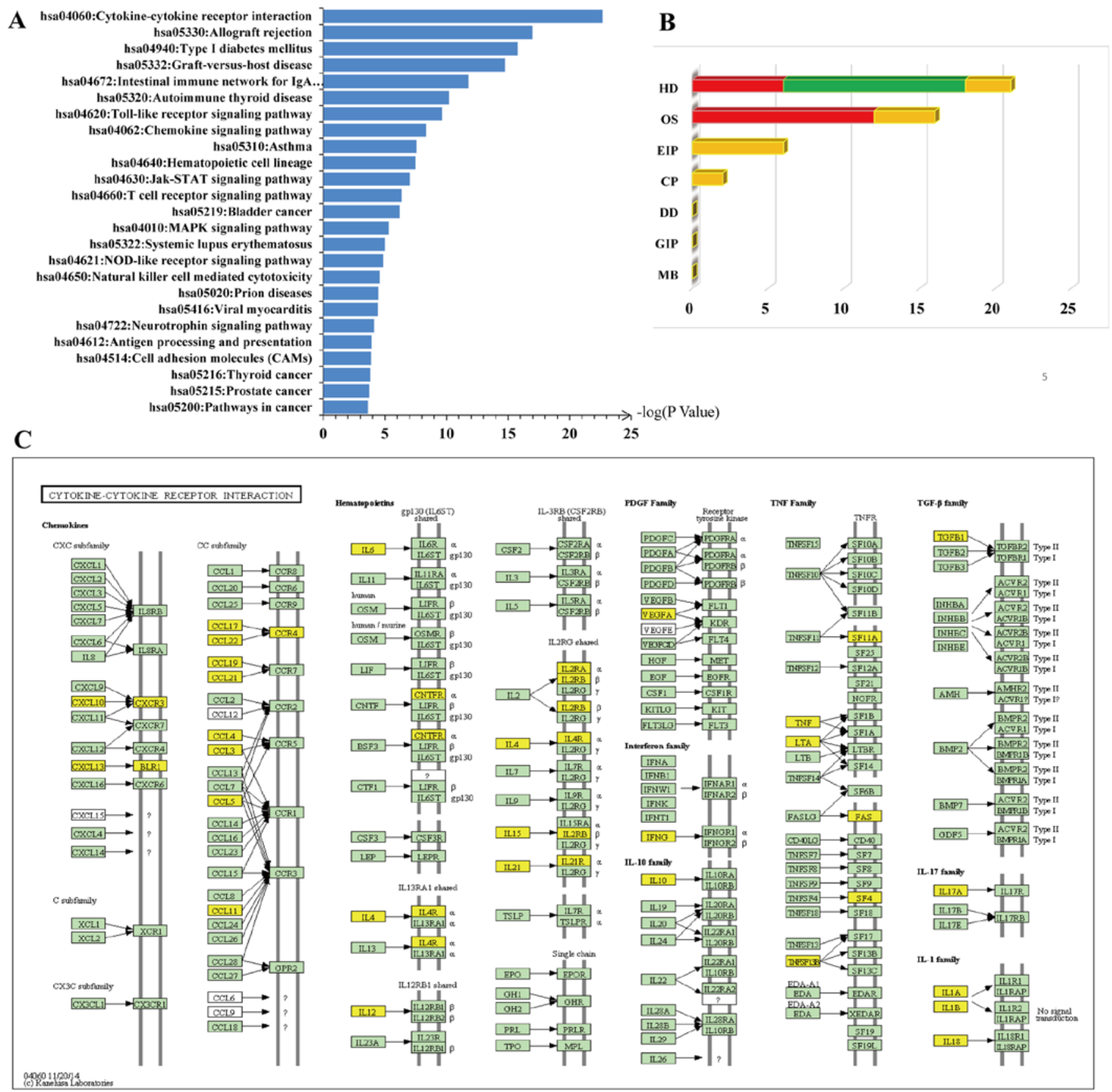

Figure 1. Myasthenia gravis (MG) risk pathways. (A) Top 25 Kyoto Encyclopedia of Genes and Genomes (KEGG) pathways enriched by MG risk genes $(\mathrm{P}<0.05)$. (B) Classification of MG risk pathways. Red represents the pathways related to immune diseases and the immune system; green represents the pathways related to cancer diseases; yellow represents other diseases. (C) Dissection of one of the MG risk pathways (hsa04060: cytokine-cytokine receptor interaction). Proteins coded by MG risk genes are indicated with a yellow background.

and $256 \mathrm{GO}$ terms were obtained (FDR value $<0.05)$, including 236 biological processes (BP), 7 molecular functions (MF) and 13 cellular components (CC) (data not shown). MG risk genes are involved in significant BP, such as immune responses, positive regulation of immune system process, regulation of cell activation and proliferation, and inflammatory responses, which conforms with the immune pathogenesis of MG.

In addition, a catalog of $85 \mathrm{MG}$ risk miRNAs was manually constructed. Among these miRNAs, 78 miRNAs and their 4,514 experimentally validated targets, as well as 12,575 miRNA-target interaction pairs, were collected. We identi- fied miRNA-regulated risk pathways and constructed an miRNA-pathway regulating network for MG. In this network, miRNA-146a regulated the most MG risk pathways (data not shown), which suggested that miRNA-146a plays a significant role in the pathologic pathways of MG. Williams et al concluded that miRNA-146a is related to immune responses and inflammatory diseases through negatively regulating immune genes, such as IL-8 and RANTES (32). Zhang et al demonstrated that silencing miRNA-146a can improve clinical myasthenic symptoms in EAMG (33). Our results were consistent with previous studies. 

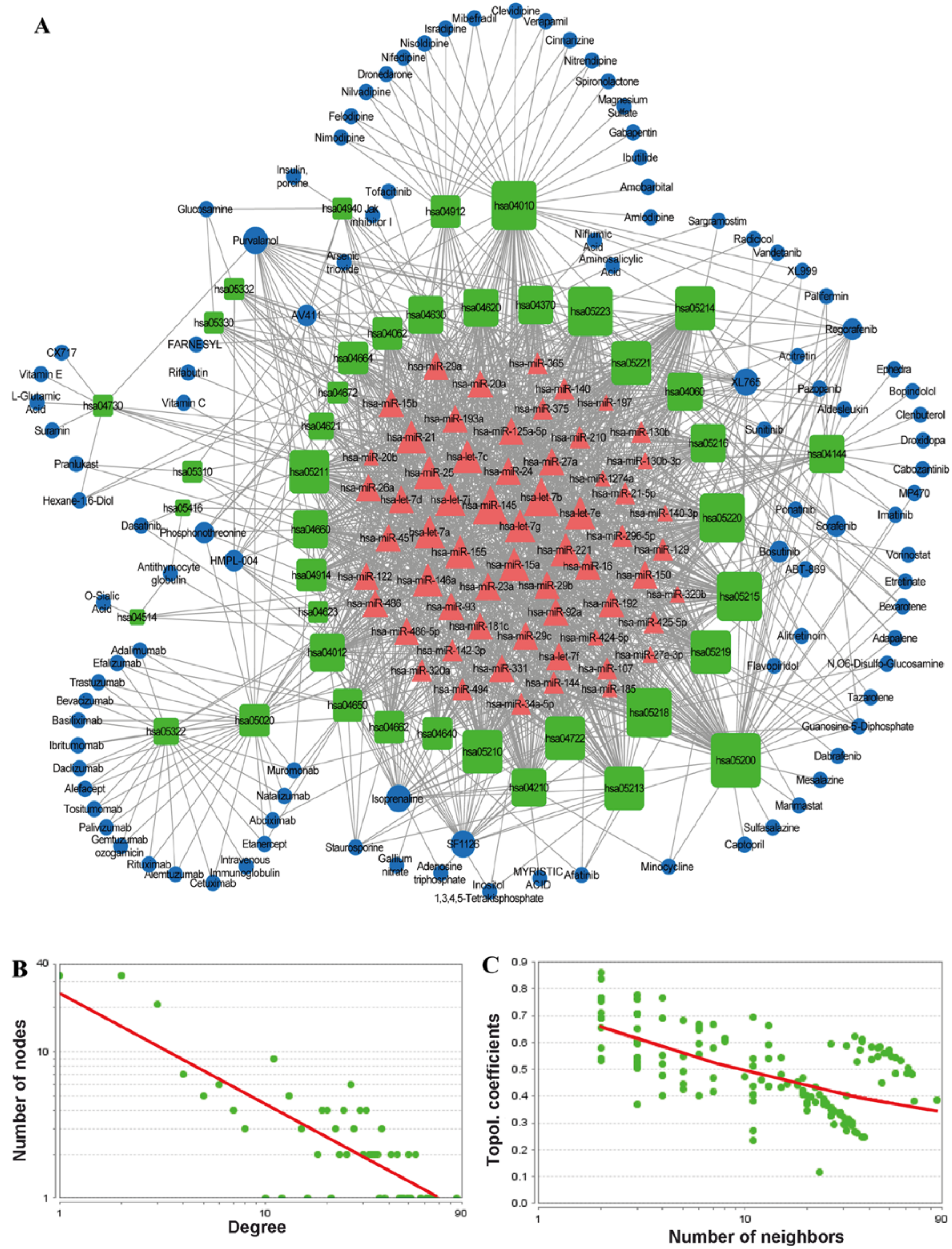

Figure 2. (A) miRNA-regulated drug-pathway network (MDPN) in myasthenia gravis (MG). Network organization of drug and pathway associations, as well as pathway and miRNA associations. Red triangles represent miRNAs, and green squares and blue ellipses indicate pathways and drugs, respectively. (B) Degree of distribution for all nodes in the MDPN. (C) Topological coefficients for all nodes in the MDPN. 

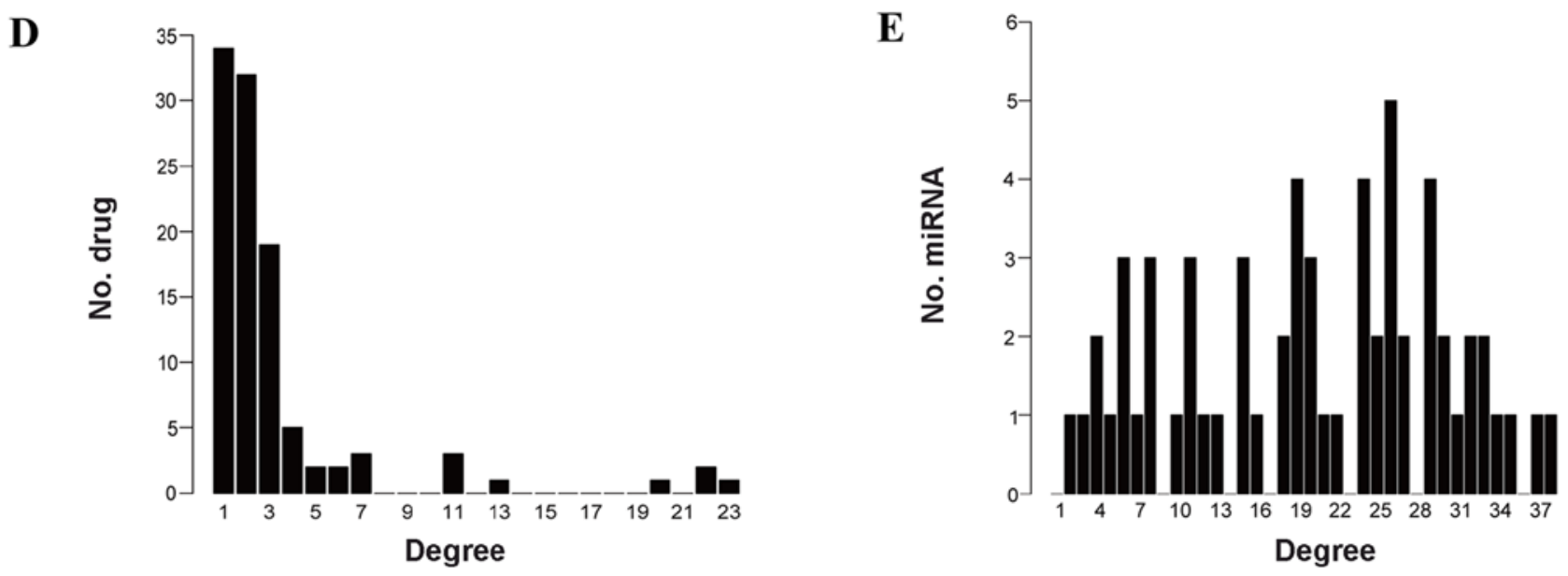

Figure 2. Continued. (D) Degree distribution of the drug nodes. (E) Degree distribution of the miRNA nodes.

Topological features of the MDPN. First, we obtained 891 drugs, 1,871 drug targets and 10,037 drug-target pairs from DrugBank. We then identified the statistically significant pathways of each drug based on its target genes. Second, we extracted drugs if their pathways overlapped with the pathways enriched by the MG risk genes and miRNA targets. Finally, we obtained 105 drugs that shared pathways with MG risk genes and miRNAs. Based on these drug-pathway and miRNA-pathway associations, we constructed an MDPN (Fig. 2A). The network contained 207 nodes and 1,575 interactions, which included 41 pathways, $61 \mathrm{MG}$ risk miRNAs and 105 drugs.

We determined the topological characteristics of the network, including the degree distribution and topological coefficient. The degree distribution followed a power law distribution $\left[f_{(x)}=25.051 \mathrm{x}^{-0.757}\right]$ using all of the nodes in the MDPN (Fig. 2B). The topological coefficient was computed to measure the extent to which a node shared links with the others in a network. As shown in Fig. 2C, with an increase in the node degree, the topological coefficient decreased. We also analyzed the degree distribution of drugs and miRNAs. The degree distribution of the drugs is displayed in Fig. 2D, which shows that the majority $(92 / 105,87.62 \%)$ of the drugs were connected by only a few pathways (no more than five). However, the degree distribution of the miRNAs was different, ranging from 2 to 37, which is displayed in Fig. 2E. There were few miRNAs associated with a small number of drugs, suggesting that these miRNAs may perturb multiple drug-related pathways and supporting the idea that miRNAs may be associated with drug sensitivity or resistance.

miRNAs and drugs synergistically regulate key MG pathways. Hsa05200 (pathway in cancer) was found to be regulated by the largest number of miRNAs and drugs after calculating the number of miRNAs and drugs for each pathway. There were $20 \mathrm{MG}$ risk genes participating in the pathway (including HRAS, BRAF, IGF1, IL-6, BCL2, MMP2, MMP9, MAPK1, FAS, VEGF, HSP90B1, PTEN, AR, TGFB1, NRAS, MAX, IGF1R, KRAS, BAX and MYC) which indicated that this pathway is extremely important in MG pathogenesis and treatment. Therefore, we performed an in-depth dissection of this pathway and identified key genes co-regulated by MG risk miRNAs and drugs (Fig. 3). We found that many MG risk genes were targeted by drugs and miRNAs, indicating that miRNAs and drugs can synergistically regulate MG risk pathways by regulating these genes. There were five MG risk genes (RAS, RAF, MMPs, VEGF and IL-6) regulated by multiple drugs and miRNAs. For example, RAS was targeted by two miRNAs (let-7b and miR-494) and a drug [guanosine-5'-diphosphate (GDP)]. RAF was targeted by 11 miRNAs (let-7 family and miR-20a, miR-145, miR-221 and miR-192) and two drugs (regorafenib and sorafenib). Arimori and Song investigated the expression of RAS and RAF and found increased levels in the peripheral blood mononuclear cells of $\mathrm{MG}$ patients that were related to clinical exacerbations of MG (34). Moreover, RAS has been previously investigated as a popular drug target related to drug sensitivity or resistance (35).

Identification of novel drug repurposing candidates for $M G$. We performed a drug repurposing strategy and identified $25 \mathrm{drug}$ repurposing candidates for $\mathrm{MG}$ with $\mathrm{Z}$ scores $>1.96(\mathrm{P}<0.05)$ (Materials and methods). Clinical trials (www.ClinicalTrials. gov) and relevant scientific literature were investigated to assess the validity of the MG repurposing candidates. Among these 25 drugs, two predicted drugs (rituximab, intravenous immunoglobulin) were studied in MG clinical studies. In addition, there were three clinic trials that studied the treatment effects of rituximab on MG, especially refractory MG (ClinicalTrials. gov identifier: nos. NCT00619671, NCT02110706 and NCT00774462). There were five clinic trials connecting intravenous immunoglobulin (IVIG) and MG (ClinicalTrials.gov identifier: nos. NCT00306033, NCT00515450, NCT01179893, NCT00004682 and NCT00774462). We also identified the relationship between the candidate drugs and MG using PubMed. Among the 25 drugs, 19 drugs $(76.0 \%)$ were directly related to the treatment of MG and/or other immune diseases. For example, adalimumab and etanercept have been investigated for treating MG. Rituximab is an anti-CD20 monoclonal antibody that can decrease plasma cells $\left(\mathrm{CD} 19^{+}\right)$and $\mathrm{B}$ cells $\left(\mathrm{CD} 20^{+}\right)$and thus suppress the production of antibodies (36). Díaz-Manera et al demonstrated that rituximab can produce long-lasting clinical benefits in patients with severe, drug-resistant MG (37). Studies have found that rituximab is beneficial for patients with MuSK antibody-positive MG (38) and can be a successful treatment 


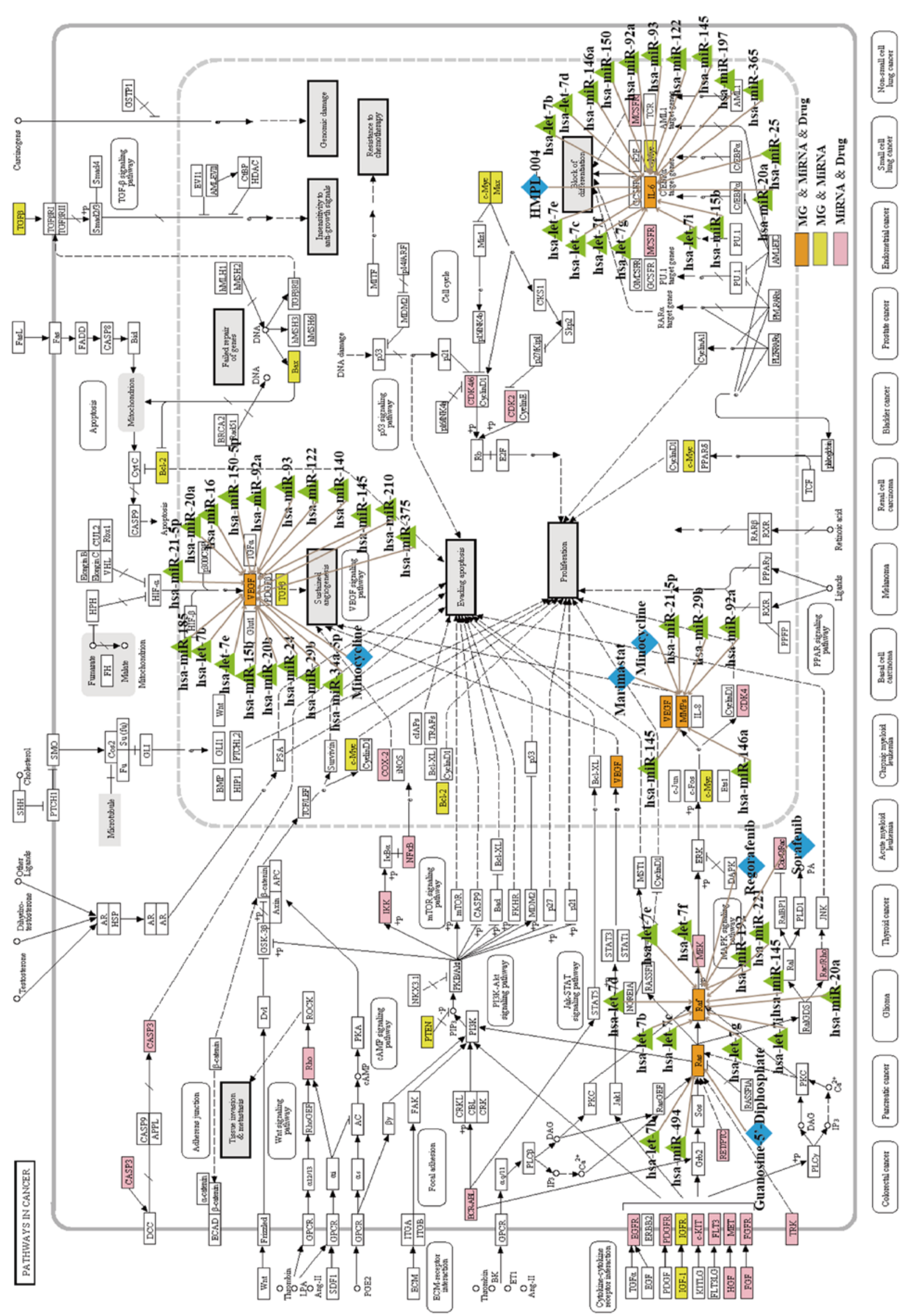

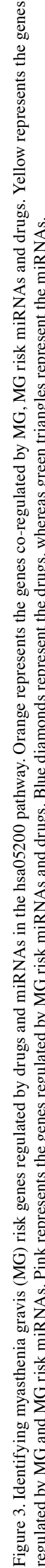


Table I. Description of the 13 candidate drugs and their related references.

\begin{tabular}{ll}
$\begin{array}{l}\text { Drug } \\
\text { candidates }\end{array}$ & Description \\
\hline Alemtuzumab & $\begin{array}{l}\text { Used for the treatment of } \\
\text { relapsing-remitting multiple } \\
\text { sclerosis and rheumatoid arthritis. }\end{array}$
\end{tabular}

Bevacizumab Bevacizumab has been reported to induce remission of psoriasis and psoriatic arthritis. Bevacizumab has been reported to ameliorate vascular and T-cell responses during experimental autoimmune encephalomyelitis.

Cetuximab Cetuximab has been reported to promote remission of rheumatoid arthritis.

Trastuzumab Trastuzumab has been reported to be used in inflammatory breast cancer.

Efalizumab Used for the treatment of plaque psoriasis and Crohn's Disease.

Insulin Used for the treatment of type 1 diabetes, which is considered an immune disease.

Gemtuzumab Used for the treatment of ozogamicin acute myeloid leukemia, which is a cancer of immune system.

Basiliximab Basiliximab has been reported to promote prolonged remission of ulcerative colitis.

Palivizumab Palivizumab has been reported to inhibit RSV-induced neurogenic-mediated inflammation.

Muromonab Muromonab is used for the prevention of organ rejection.

Abciximab Abciximab is used to alleviate vascular inflammation caused by both acute coronary syndromes and injury after percutaneous coronary intervention.

Tositumomab Used for the treatment of non-Hodgkin's lymphoma, which is a cancer of immune system.

Ibritumomab Used for the treatment of non-Hodgkin's lymphoma, which is a cancer of immune system.

Refs.

for refractory generalized MG (39). Sunitinib has been reported to improve MG when it appears with thymomas (40). Another 13 candidate drugs were reported to treat other immune and inflammatory diseases, such as rheumatoid arthritis, psoriatic arthritis, psoriasis and multiple sclerosis, supporting their potential use in MG (Table I).

Mechanism dissection of novel drug candidates and miRNAs in $M G$. To illustrate the complex relationship and underlying mechanisms among drugs, drug targets, $\mathrm{MG}$ risk genes, $\mathrm{MG}$ risk miRNAs and pathways, we constructed a layered network that can provide a convenient way for investigating detailed information regarding these associations. The links between every two layers were as follows: i) drug action on drug targets; ii) the influence of drug targets on MG risk pathways; iii) the effect of pathways on MG risk genes; iv) MG risk miRNA targets on MG risk genes; v) MG risk gene association with MG. We constructed the layered network between rituximab and $\mathrm{MG}$ to reveal the potential treatment effects of rituximab in MG (Fig.4). Two pathways (hsa05322: systemic lupus erythematosus and hsa05020: prion diseases) were inferred, connecting rituximab and MG. These two pathways influenced MG risk genes, mainly IL-6, IL-1A, IL-1B, MAPK1, tumor necrosis factor (TNF), BAX and IFNG. The gene IL-6, which encodes a cytokine that functions in inflammation and the maturation of B cells, was targeted by MG-related miRNAs, such as let 7 family, miR-150, miR-145, miR-122, miR-421 and miR-25 (Fig. 4). The gene MAPK1 encodes a member of the MAP kinase family and is an integration point for multiple biochemical signals, which are involved in a variety of cellular processes, such as proliferation, differentiation and development. MAPK1 was targeted by miR-25, miR-129, miR-494, miR-15b, miR-24, miR320a and miR-146a. The TNF gene encodes a multifunctional pro-inflammatory cytokine that belongs to the tumor necrosis factor superfamily and is targeted by the let 7 family, miR-16, miR-363, miR-25, miR-20a, miR-122, miR-140, miR-150 and miR-146a. This suggested that the function of genes can be influenced by abnormal expression of disease-related miRNAs. Similarly, we constructed a layered network among adalimumab (Fig. 5), sunitinib (Fig. 6), muromonab (Fig. 7) and MG. Sunitinib and ABT-869 targeted the same pathways, including hsa04060 (cytokine-cytokine receptor interaction), hsa04144 (endocytosis), hsa04640 (hematopoietic cell lineage) and hsa05200 (pathway in cancer), indicating that the two drugs can function through similar pathways, again providing evidence for drug combination.

\section{Discussion}

MG is a rare disease, and the current treatments for this disease do not meet the expectations of affected patients. Oral cholinesterase inhibitors, which can inhibit the activity of the enzyme acetylcholinesterase (AChE) and increase the level of acetylcholine available for binding at the $\mathrm{NMJ}$, are the first-line treatment in patients with MG (14). However, AChE inhibitors rarely induce complete relief of $\mathrm{MG}$ symptoms and barely affect disease progression. Plasma exchange (PE) and intravenous immunoglobulin are used for the treatment of MG exacerbations to achieve a rapid clinical response by reducing the concentrations of circulating antibodies (41). However, both therapies are expensive and can induce side-effects, including hypotension and paresthesias from citrate-induced hypocalcaemia. Corticosteroids (CSs) were the first immune-suppressants (ISs) 


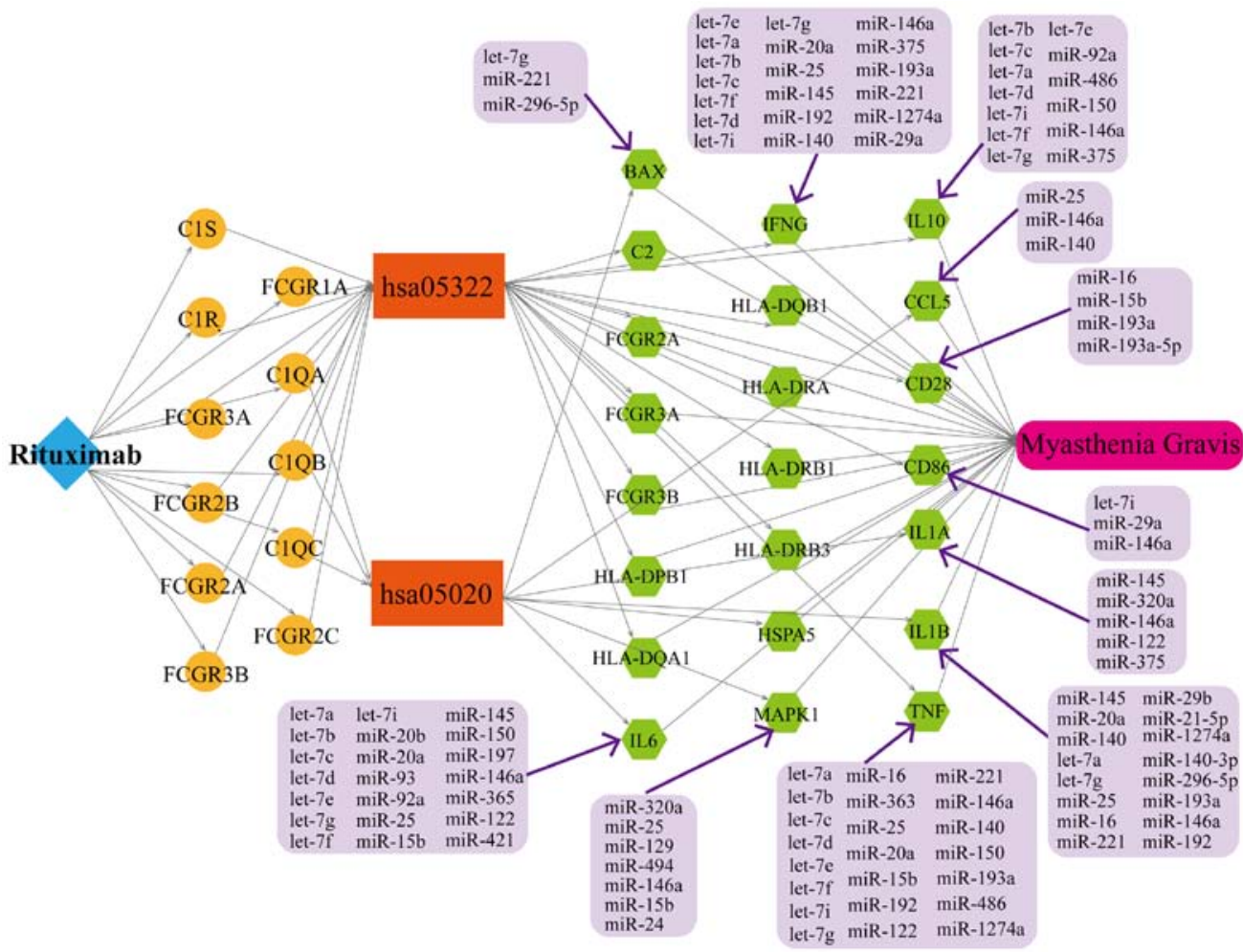

Figure 4. Layered network between rituximab and myasthenia gravis (MG). Diamond indicates drug, circles indicate drug target genes, rectangles indicate the MG risk pathways, and hexagons indicate MG risk genes that regulate the MG risk pathway. Many MG risk genes carry validated miRNA targets.

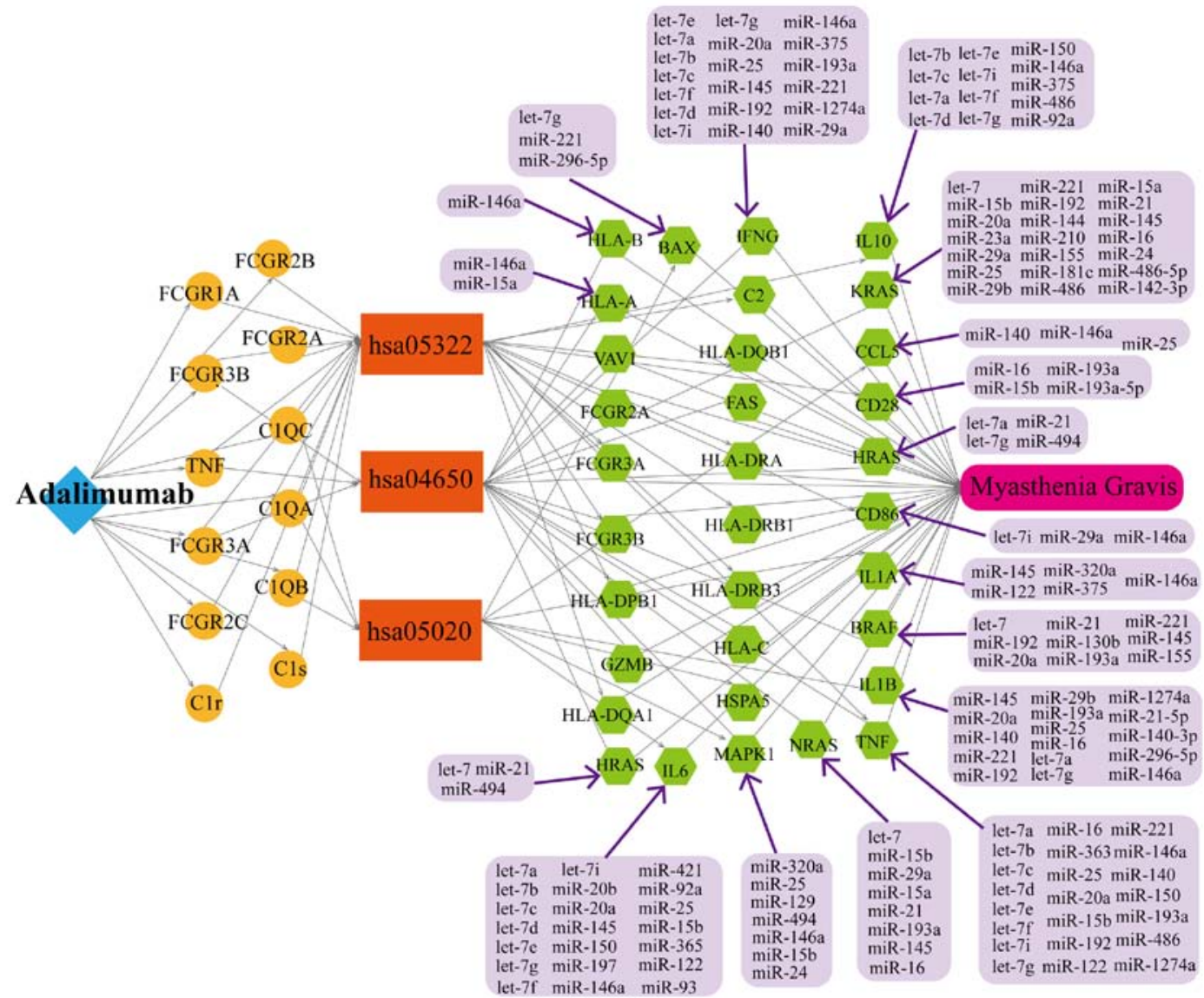

Figure 5. Layered network between adalimumab and MG. Diamond indicates drug, circles indicate drug target genes, rectangles indicate the MG risk pathways, and hexagons indicate MG risk genes that regulate the MG risk pathway. Many MG risk genes carry validated miRNA targets. 


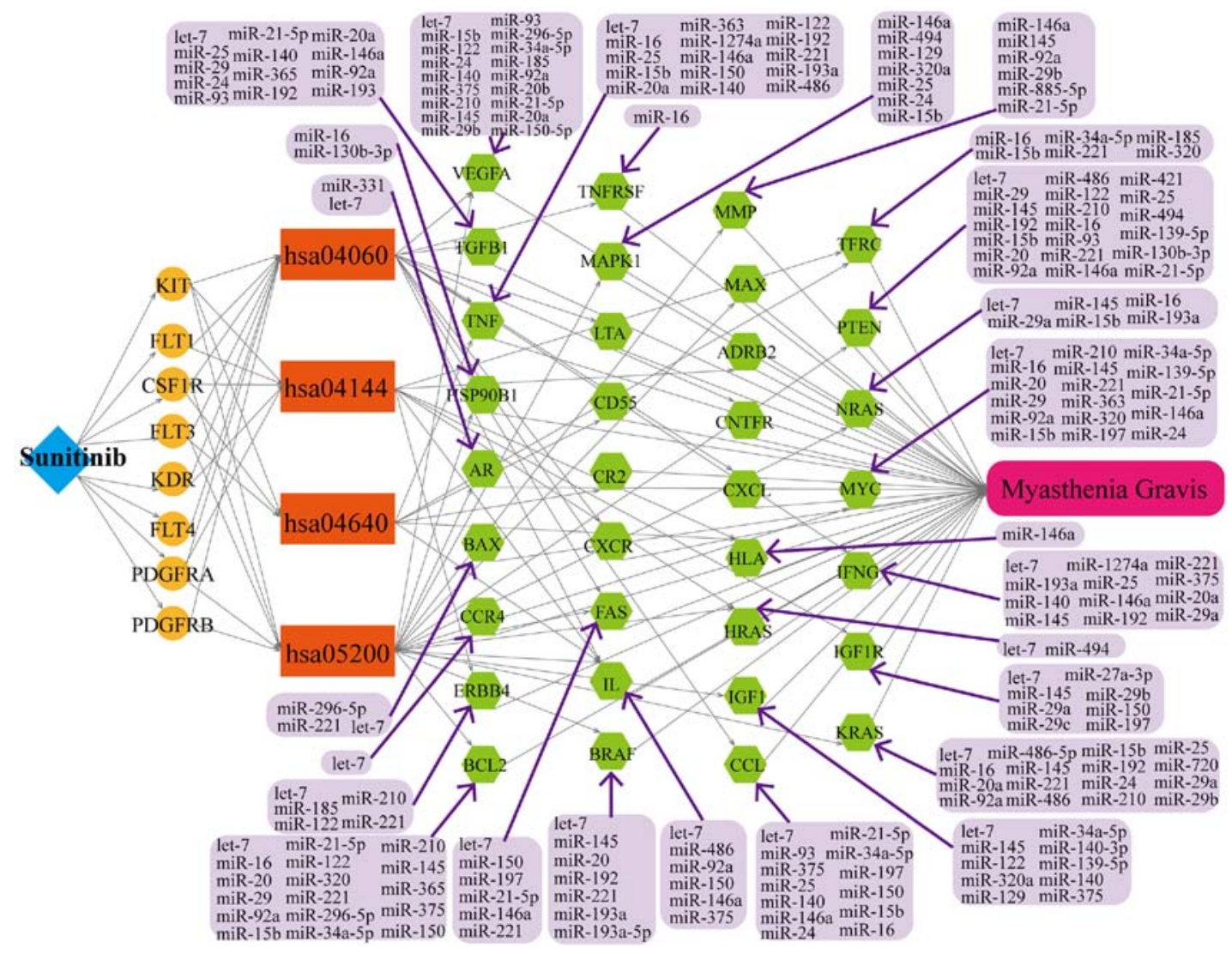

Figure 6. Layered network between sunitinib and myasthenia gravis (MG). Diamond indicates drug, circles indicate drug target genes, rectangles indicate the MG risk pathways, and hexagons indicate MG risk genes that regulate the MG risk pathway. Many MG risk genes carry validated miRNA targets.

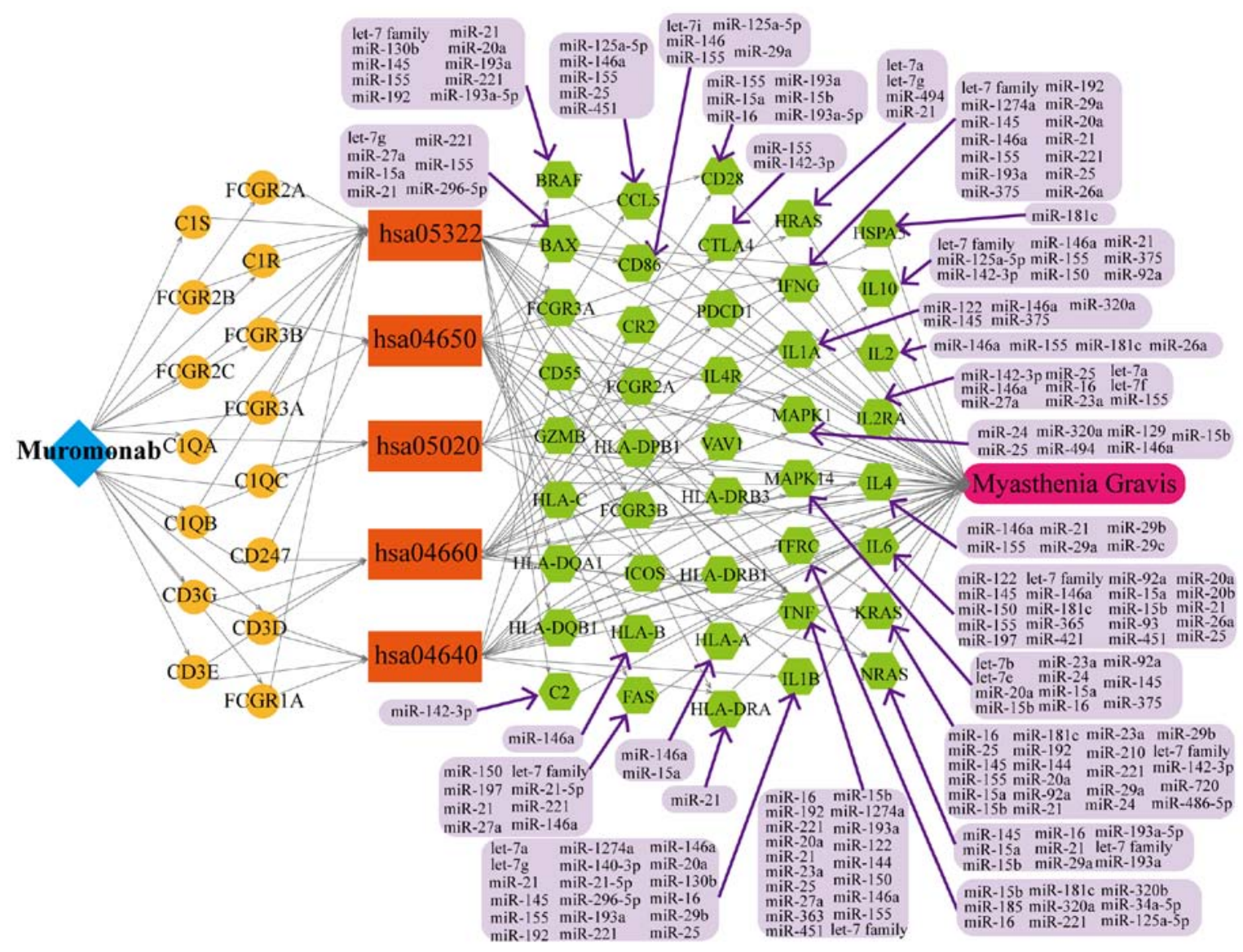

Figure 7. Layered network between muromonab and myasthenia gravis (MG). Diamond indicates drug, circles indicate drug target genes, rectangles indicate the MG risk pathways, and hexagons indicate MG risk genes that regulate the MG risk pathway. Many MG risk genes carry validated miRNA targets. 
to be used in MG and remain the most commonly used immunetargeted therapy. However, common side-effects include Cushing's syndrome, osteoporosis, weight gain, hyperglycemia, diabetes, hypertension, gastritis or ulcers (4). Therefore, there is an urgent need for effective drugs that hit new MG targets.

In this study, we comprehensively selected MG risk genes and miRNAs and identified the MG risk pathways enriched by these genes and miRNAs. The MG risk pathways were mainly related to immunity and neoplasms, which may explain why MG sometimes occurs as a paraneoplastic syndrome associated with thymoma (42). Then, we identified drugs that targeted risk pathways with MG risk genes and miRNAs. We also analyzed how the miRNAs and drugs synergistically regulated MG risk pathways. We constructed an miRNA-regulated drug-pathway network of MG, which can help us understand the risk pathways regulated by MG risk miRNAs and drugs. Our method comprehensively considers the common pathways between drugs and $\mathrm{MG}$ rather than their common targets. Among the 25 drug repurposing candidates, $19(76.0 \%)$ were related to MG or other immune diseases, supporting by clinical trials or related literature. Kim et al (14) identified treatments for MG based on its immune pathogenesis and suggested that treatment of $\mathrm{MG}$ should be according to the clinical features or subtypes of the disease, the severity of the disease and the activities of daily living of individual patients. Lewis (15) identified new potential immunotherapies for MG through the literature mining current treatments for immune diseases. Rituximab can target B cells by directly targeting CD20 molecules, which is consistent with our results. Whereas Lewis (15) and Kim et al (14) considered the pathology of MG based on its immune aspects, our study was based on the pathways that were enriched by MG risk genes and miRNAs.

The risk pathways of MG not only featured immune aspects but were also related to cancer and other diseases. It is unreasonable to characterize this disease from only an immune aspect; treatment based on immune pathology is not sufficient, which may explain why some patients do not respond to immune therapy. Therefore, identifying repurposing candidate drugs for MG based on the common pathways and drugs associated with this disease is more comprehensive and reliable. To determine whether these repurposing candidate drugs are applicable for the treatment of MG, well-designed experiments or clinical trials should be conducted.

In conclusion, we comprehensively collected the risk genes and miRNAs of MG and identified MG risk pathways to characterize the mechanism of and treatment strategy for MG. We also constructed an MDPN to better understand the relationship among genes, pathways, and drugs and to identify promising drug repurposing candidates for MG.

\section{Acknowledgements}

This study was supported by the National Natural Science Foundation of China (grant nos. 81371324, 81571166 and 31401090), the Specialized Research Fund for the Doctoral Program of Higher Education of China (grant no. 20132307110008), and the Foundation of Heilongjiang Medical Science Institute (no. 201406). In addition, we would like to thank Nature Publishing Group (http://languageediting. nature.com/editing-services) for English language editing.

\section{References}

1. Meriggioli MN and Sanders DB: Autoimmune myasthenia gravis: Emerging clinical and biological heterogeneity. Lancet Neurol 8: 475-490, 2009.

2. Juel VC and Massey JM: Myasthenia gravis. Orphanet J Rare Dis 2: 44, 2007.

3. Zisimopoulou P, Evangelakou P, Tzartos J, Lazaridis K, Zouvelou V, Mantegazza R, Antozzi C, Andreetta F, Evoli A, Deymeer F, et al: A comprehensive analysis of the epidemiology and clinical characteristics of anti-LRP4 in myasthenia gravis. J Autoimmun 52: 139-145, 2014.

4. Yeh JH, Chen HJ, Chen YK, Chiu HC and Kao CH: Increased risk of osteoporosis in patients with myasthenia gravis: A population-based cohort study. Neurology 83: 1075-1079, 2014.

5. Cheng Z, Qiu S, Jiang L, Zhang A, Bao W, Liu P and Liu J: MiR-320a is downregulated in patients with myasthenia gravis and modulates inflammatory cytokines production by targeting mitogen-activated protein kinase 1. J Clin Immunol 33: 567-576, 2013.

6. Lu J, Yan M, Wang Y, Zhang J, Yang H, Tian FF, Zhou W, Zhang N and Li J: Altered expression of miR-146a in myasthenia gravis. Neurosci Lett 555: 85-90, 2013.

7. Zhang Y, Guo M, Xin N, Shao Z, Zhang X, Zhang Y, Chen J, Zheng S, Fu L, Wang Y, et al: Decreased microRNA miR-181c expression in peripheral blood mononuclear cells correlates with elevated serum levels of IL-7 and IL-17 in patients with myasthenia gravis. Clin Exp Med 16: 413-421, 2016.

8. Garzon R, Marcucci G and Croce CM: Targeting microRNAs in cancer: Rationale, strategies and challenges. Nat Rev Drug Discov 9: 775-789, 2010.

9. Wang YZ, Tian FF, Yan M, Zhang JM, Liu Q, Lu JY, Zhou WB, Yang $\mathrm{H}$ and Li J: Delivery of an miR155 inhibitor by anti-CD20 single-chain antibody into $\mathrm{B}$ cells reduces the acetylcholine receptor-specific autoantibodies and ameliorates experimental autoimmune myasthenia gravis. Clin Exp Immunol 176: 207-221, 2014.

10. Ashburn TT and Thor KB: Drug repositioning: Identifying and developing new uses for existing drugs. Nat Rev Drug Discov 3: 673-683, 2004.

11. Hu G and Agarwal P: Human disease-drug network based on genomic expression profiles. PLoS One 4: e6536, 2009.

12. Meng F, Dai E, Yu X, Zhang Y, Chen X, Liu X, Wang S, Wang L and Jiang W: Constructing and characterizing a bioactive small molecule and microRNA association network for Alzheimer's disease. J R Soc Interface 11: 20131057, 2013.

13. Ye H, Yang LL, Cao ZW, Tang KL and Li YX: A pathway profile-based method for drug repositioning. Chin Sci Bull 57: 2106-2112, 2012.

14. Kim JY, Park KD and Richman DP: Treatment of myasthenia gravis based on its immunopathogenesis. J Clin Neurol 7: 173-183, 2011.

15. Lewis RA: Myasthenia gravis: New therapeutic approaches based on pathophysiology. J Neurol Sci 333: 93-98, 2013.

16. Yang L, Wang J, Sun X, Cao Y, Ning S, Zhang H, Chen L, Li R, Tian Q, Wang L, et al: Identifying a polymorphic 'switch' that influences miRNAs' regulation of a myasthenia gravis risk pathway. PLoS One 9: e104827, 2014.

17. Becker KG, Barnes KC, Bright TJ and Wang SA: The genetic association database. Nat Genet 36: 431-432, 2004.

18. Piñero J, Queralt-Rosinach N, Bravo À, Deu-Pons J, Bauer-Mehren A, Baron M, Sanz F and Furlong LI: DisGeNET: a discovery platform for the dynamical exploration of human diseases and their genes. Database (Oxford) 2015: bav028, 2015.

19. McKusick VA: Mendelian inheritance in man and its online version, OMIM. Am J Hum Genet 80: 588-604, 2007.

20. Schriml LM, Arze C, Nadendla S, Chang YW, Mazaitis M, Felix V, Feng G and Kibbe WA: Disease Ontology: A backbone for disease semantic integration. Nucleic Acids Res 40: D940-D946, 2012.

21. Li Y, Qiu C, Tu J, Geng B, Yang J, Jiang T and Cui Q: HMDD v2.0: A database for experimentally supported human microRNA and disease associations. Nucleic Acids Res 42: D1070-D1074, 2014.

22. Ruepp A, Kowarsch A, Schmidl D, Buggenthin F, Brauner B, Dunger I, Fobo G, Frishman G, Montrone C and Theis FJ: PhenomiR: A knowledgebase for microRNA expression in diseases and biological processes. Genome Biol 11: R6, 2010.

23. Dweep H, Sticht C, Pandey P and Gretz N: miRWalk - database: Prediction of possible miRNA binding sites by 'walking' the genes of three genomes. J Biomed Inform 44: 839-847, 2011. 
24. Hsu SD, Tseng YT, Shrestha S, Lin YL, Khaleel A, Chou CH, Chu CF, Huang HY, Lin CM, Ho SY, et al: miRTarBase update 2014: An information resource for experimentally validated miRNA-target interactions. Nucleic Acids Res 42: D78-D85, 2014.

25. Jiang Q, Wang Y, Hao Y, Juan L, Teng M, Zhang X, Li M, Wang $G$ and Liu Y: miR2Disease: A manually curated database for microRNA deregulation in human disease. Nucleic Acids Res 37: D98-D104, 2009.

26. Xiao F, Zuo Z, Cai G, Kang S, Gao X and Li T: miRecords: An integrated resource for microRNA-target interactions. Nucleic Acids Res 37: D105-D110, 2009.

27. Naeem H, Küffner R, Csaba G and Zimmer R: miRSel: Automated extraction of associations between microRNAs and genes from the biomedical literature. BMC Bioinformatics 11: 135, 2010.

28. Law V, Knox C, Djoumbou Y, Jewison T, Guo AC, Liu Y, Maciejewski A, Arndt D, Wilson M, Neveu V, et al: DrugBank 4.0: Shedding new light on drug metabolism. Nucleic Acids Res 42: D1091-D1097, 2014.

29. Dennis G Jr, Sherman BT, Hosack DA, Yang J, Gao W, Lane HC and Lempicki RA: DAVID: Database for Annotation, Visualization, and Integrated Discovery. Genome Biol 4: 3, 2003.

30. Smoot ME, Ono K, Ruscheinski J, Wang PL and Ideker T: Cytoscape 2.8: New features for data integration and network visualization. Bioinformatics 27: 431-432, 2011.

31. Jebb A, Hadden P and Broom RJ: Uveal metastasis and myasthenia gravis in a patient with recurrent renal cell carcinoma treated with pazopanib. Clin Genitourin Cancer 12: e143-e146, 2014.

32. Williams AE, Perry MM, Moschos SA, Larner-Svensson HM and Lindsay MA: Role of miRNA-146a in the regulation of the innate immune response and cancer. Biochem Soc Trans 36: 1211-1215, 2008

33. Zhang J, Jia G, Liu Q, Hu J, Yan M, Yang B, Yang H, Zhou W and Li J: Silencing miR-146a influences B cells and ameliorates experimental autoimmune myasthenia gravis. Immunology 144: $56-67,2015$.

34. Arimori S and Song ZJ: Expression of c-myc, c-Ki-ras and c-Ha-ras oncogene products in peripheral blood mononuclear cells from patients with myasthenia gravis. Intern Med 32: 519-522, 1993.

35. Aznar S and Lacal JC: Searching new targets for anticancer drug design: The families of Ras and Rho GTPases and their effectors Prog Nucleic Acid Res Mol Biol 67: 193-234, 2001.

36. Palanichamy A, Jahn S, Nickles D, Derstine M, Abounasr A, Hauser SL, Baranzini SE, Leppert D and von Büdingen HC: Rituximab efficiently depletes increased CD20-expressing T cells in multiple sclerosis patients. J Immunol 193: 580-586, 2014.

37. Díaz-Manera J, Martínez-Hernández E, Querol L, Klooster R, Rojas-García R, Suárez-Calvet X, Muñoz-Blanco JL, Mazia C, Straasheijm KR, Gallardo E, et al: Long-lasting treatment effect of rituximab in MuSK myasthenia. Neurology 78: 189-193, 2012.

38. Stein B and Bird SJ: Rituximab in the treatment of MuSK antibody-positive myasthenia gravis. J Clin Neuromuscul Dis 12: 163-164, 2011.

39. Lebrun C, Bourg V, Tieulie N and Thomas P: Successful treatment of refractory generalized myasthenia gravis with rituximab. Eur J Neurol 16: 246-250, 2009.

40. Thomas A, Rajan A, Berman A, Tomita Y, Brzezniak C, Lee MJ, Lee S, Ling A, Spittler AJ, Carter CA, et al: Sunitinib in patients with chemotherapy-refractory thymoma and thymic carcinoma: an open-label phase 2 trial. Lancet Oncol 16: 177-186, 2015.

41. Samuelsson A, Towers TL and Ravetch JV: Anti-inflammatory activity of IVIG mediated through the inhibitory Fc receptor. Science 291: 484-486, 2001.

42. Qiao J, Zhou G, Ding Y, Zhu D and Fang H: Multiple paraneoplastic syndromes: Myasthenia gravis, vitiligo, alopecia areata, and oral lichen planus associated with thymoma. J Neurol Sci 308: 177-179, 2011.

43. Arroyo González R, Kita M, Crayton H, Havrdova E, Margolin DH, Lake SL, Giovannoni G; CARE-MS I and II Investigators: Alemtuzumab improves quality-of-life outcomes compared with subcutaneous interferon beta-la in patients with active relapsingremitting multiple sclerosis. Mult Scler: Nov 24, 2016 (Epub ahead of print).
44. Giovannoni G, Cohen JA, Coles AJ, Hartung HP, Havrdova E, Selmaj KW, Margolin DH, Lake SL, Kaup SM, Panzara MA, Compston DA; CARE-MS II Investigators: Alemtuzumab improves preexisting disability in active relapsing-remitting MS patients. Neurology 87: 1985-1992, 2016.

45. Lorenzi AR, Clarke AM, Wooldridge T, Waldmann H, Hale G, Symmons D, Hazleman BL and Isaacs JD: Morbidity and mortality in rheumatoid arthritis patients with prolonged therapy-induced lymphopenia: twelve-year outcomes. Arthritis Rheum 58: 370-375, 2008.

46. Datta-Mitra A, Riar NK and Raychaudhuri SP: Remission of psoriasis and psoriatic arthritis during bevacizumab therapy for renal cell cancer. Indian J Dermatol 59: 632, 2014.

47. Akman A, Yilmaz E, Mutlu H and Ozdogan M: Complete remission of psoriasis following bevacizumab therapy for colon cancer. Clin Exp Dermatol 34: e202-e204, 2009.

48. MacMillan CJ, Furlong SJ, Doucette CD, Chen PL, Hoskin DW and Easton AS: Bevacizumab diminishes experimental autoimmune encephalomyelitis by inhibiting spinal cord angiogenesis and reducing peripheral T-cell responses. J Neuropathol Exp Neurol 71: 983-999, 2012.

49. Sullivan T, Benjamin CG, Kempf PW and Deeken JF: Cetuximab in the treatment of rheumatoid arthritis. J Clin Rheumatol 16 : 32-33, 2010

50. Dawood S, Gong Y, Broglio K, Buchholz TA, Woodward W, Lucci A, Valero V, Gonzalez-Angulo AM, Hortobagyi GN and Cristofanilli M: Trastuzumab in primary inflammatory breast cancer (IBC): High pathological response rates and improved outcome. Breast J 16: 529-532, 2010.

51. Lotti T, Chimenti S, Katsambas A, Ortonne JP, Dubertret L, Licu D and Simon J: Efficacy and safety of efalizumab in patients with moderate-to-severe plaque psoriasis resistant to previous anti-psoriatic treatment: Results of a multicentre, open-label, phase IIIb/IV trial. Arch Drug Inf 3: 9-18, 2010.

52. James DG, Seo DH, Chen J, Vemulapalli C and Stone CD: Efalizumab, a human monoclonal anti-CD11a antibody, in the treatment of moderate to severe Crohn's disease: an open-label pilot study. Dig Dis Sci 56: 1806-1810, 2011.

53. Polonsky W, Traylor L, Gao L, Wei W, Ameer B, Stuhr A and Vlajnic A: Improved treatment satisfaction in patients with type 1 diabetes treated with insulin glargine $100 \mathrm{U} / \mathrm{mL}$ versus neutral protamine Hagedorn insulin: An exploration of key predictors from two randomized controlled trials. J Diabetes Complications: Dec 8, 2016 (Epub ahead of print).

54. Cowan AJ, Laszlo GS, Estey EH and Walter RB: Antibody-based therapy of acute myeloid leukemia with gemtuzumab ozogamicin. Front Biosci (Landmark Ed) 18: 1311-1334, 2013.

55. Creed TJ, Probert CS, Norman MN, Moorghen M, Shepherd NA, Hearing SD, Dayan CM; BASBUC INVESTIGATORS: Basiliximab for the treatment of steroid-resistant ulcerative colitis: further experience in moderate and severe disease. Aliment Pharmacol Ther 23: 1435-1442, 2006.

56. Piedimonte G, King KA, Holmgren NL, Bertrand PJ, Rodriguez MM and Hirsch RL: A humanized monoclonal antibody against respiratory syncytial virus (palivizumab) inhibits RSV-induced neurogenic-mediated inflammation in rat airways. Pediatr Res 47: 351-356, 2000.

57. Ueda D, Hori T, Nguyen JH and Uemoto S: Muromonab-CD3 therapy for refractory rejections after liver transplantation: a single-center experience during two decades in Japan. J Hepatobiliary Pancreat Sci 17: 885-891, 2010.

58. Kereiakes DJ: Inflammation as a therapeutic target: a unique role for abciximab. Am Heart J 146 (Suppl 4): S1-S4, 2003.

59. Jacene H, Crandall J, Kasamon YL, Ambinder RF, Piantadosi S, Serena D, Kasecamp W and Wahl RL: Initial experience with tositumomab and I-131-labeled tositumomab for treatment of relapsed/refractory Hodgkin lymphoma. Mol Imaging Biol: Oct 31, 2016 (Epub ahead of print).

60. Arnason JE, Luptakova K, Rosenblatt J, Tzachanis D, Avigan D, Zwicker JI, Levine J, Kim M, Parker JA, Grant B and Joyce RM: Yttrium-90 ibritumomab tiuxetan followed by rituximab maintenance as treatment for patients with diffuse large B-cell lymphoma are not candidates for autologous stem cell transplant. Acta Haematol 133: 347-353, 2015. 